\title{
Phosphorus from desert dust can be directly utilized by plant leaves
}

\author{
AVNER GROSS ${ }^{1}$, SUDEEP TIWARI ${ }^{2}$, ILANA SHTEIN ${ }^{3}$ \\ AND RAN EREL ${ }^{4}$
}

${ }^{1}$ ben gurion university of the negev

${ }^{2}$ Ben Gurion University, Israel

33. Eastern Region Research and Development Center, Ariel, Israel.

${ }^{4}$ Agricultural Research Organization

Presenting Author: avnergro@bgu.ac.il

Phosphorus (P) scarcity constrains plant growth in many ecosystems worldwide. In $P$ poor ecosystems, biogeochemical theory links plant productivity with the deposition of P-rich desert dust. The traditional view is that plants acquire $P$ from soil exclusively via roots. Here we show that $P$ deficient plants acquire insoluble $P$ from desert dust directly via their leaves. Foliar $P$ uptake from desert dust doubled the growth of chickpea and wheat, two widely cultivated crops originating in the vicinity of the Syrian Desert. $P$ deficiency enabled acquisition of insoluble $P$ forms conventionally perceived as unavailable for plants. $P$ deficiency induced leaf modifications increased foliar dust capture, acidified the leaf surface and, in chickpea, enhanced exudation of $P$ solubilizing organic acids. The abovementioned foliar responses are comparable to known root responses and thus show convergent adaptations to $P$ uptake. Foliar responses did not occur in maize, which displayed only a marginal response to dust. Assuming foliar dust $P$ uptake trait is common among plant communities, we estimate the effective $P$ utilization from desert dust to be several folds higher than previously postulated, and should be incorporated into terrestrial ecosystem models and into traditional frameworks of ecosystems $P$ budgets. 